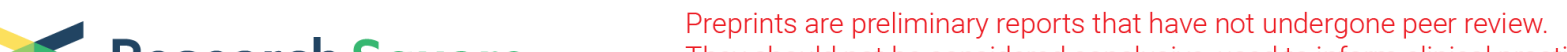 Research Square
They should not be considered conclusive, used to inform clinical practice,
or refernced by the media as validated information.
}

\section{Sheng Jing Decoction, as a Traditional Chinese Medicine Prescription, Can Promote Spermatogenesis and Increase Sperm Motility}

\section{Guang Yan}

Seventh People's Hospital of Shanghai University of TCM

\section{Fang Tian}

Fudan university

Peng Liu

Seventh People's Hospital of Shanghai University of TCM

Jianmin Mao

Seventh People's Hospital of Shanghai University of TCM

Jianming Sun ( $\nabla$ sunjianmingdoctor@163.com )

Seventh People's Hospital of Shanghai University of TCM

\section{Wenjun Han}

Seventh People's Hospital of Shanghai University of TCM

\section{Ran Mo}

Seventh People's Hospital of Shanghai University of TCM

\section{Shishuai Guo}

Seventh People's Hospital of Shanghai University of TCM

\section{Quanyao Yu}

Seventh People's Hospital of Shanghai University of TCM

\section{Research}

Keywords: Sheng Jing Decoction, SJD, spermatogenesis, sperm motility, male infertility

Posted Date: February 4th, 2021

DOI: https://doi.org/10.21203/rs.3.rs-167175/v1

License: (c) (i) This work is licensed under a Creative Commons Attribution 4.0 International License. Read Full License 


\section{Abstract}

\section{Background}

Sheng Jing Decoction (SJD), as a traditional Chinese medicine prescription, is mainly be used to treat male infertility. It has been applied in many hospitals in China, and the clinical effect of patients' reaction is satisfactory. However, pharmacological function and molecular mechanism of SJD are poorly understood. In this study, mainly investigated the function of SJD on spermatogenesis and sperm motility, and explored the potential mechanisms.

\section{Methods}

The oligozoospermia model of ICR mice was induced by injecting intraperitoneally (ip) with $60 \mathrm{mg} / \mathrm{kg}$ dose of cyclophosphamide. At the same time of modeling, high, medium and low doses of SJD were given orally for treatment respectively. Sperm vitality and motility were detected and analyzed by CASA, and sperm morphology was tested by Papanicolaou staining. Sperm mitochondrial membrane potential (MMP) was tested by JC-1 staining. Sperm plasma membrane integrity was determined by SYBR-14/PI double staining. Histopathological changes of testis were analyzed by HE staining and Immunohistochemistry. Genes expression related to spermatogenesis and sperm development were detected by real-time RT-PCR.

Results

High, medium and low doses of SJD are effective to recover from the impairment of the whole body and testicular tissue by cyclophosphamide inducing, and to rescue the damage of testicular tissue cells including sertoli cells and germ cells by cyclophosphamide inducing. SJD can all partly restore the decrease in sperm concentration, sperm vitality, sperm motility and normal sperm morphology rate by cyclophosphamide inducing. Ki67 staining analyses confirm SJD can promoted testicular tissue cells proliferation. Real-time RT-PCR analyses reveal SJD can up-regulates the expression of proliferationassociated gene Lin28a, and differentiation-associated genes Kit, Soh/h2 and Stra8. SJD can also reduce the impairment of MMP and sperm plasma membrane integrity by cyclophosphamide inducing.

Conclusion

SJD is effective to support sperm quantity and quality by increasing sperm concentration, sperm vitality, sperm motility and normal sperm morphology rate. SJD can promote spermatogenesis by up-regulating the expression of the proliferation-associated gene Lin28a, and the differentiation-associated genes (Kit, Soh/h2 and Stra8). SJD can sustain MMP and sperm plasma membrane integrity to increase sperm motility.

\section{Background}


Infertility is defined by the World Health Organization as no conception after $\geq 12$ months of regular unprotected intercourse[1]. Male infertility usually refers a male's inability to cause pregnancy in a healthy female partner. Up to $15 \%$ of couples suffer from infertility, and male infertility accounts for no fewer than $50 \%$ of infertile couples[2, 3]. Male infertility is a complex multifactorial disease affecting approximately $7 \%$ of the male population[4]. The four major aetiological categories of male infertility are spermatogenic qualitative defects, spermatogenic quantitative defects, hypothalamic-pituitary axis disturbances, and ductal obstruction or dysfunction[5]. The disorder of spermatogenesis caused by genetic and nongenetic factors contributes to spermatogenic quantitative and qualitative defects[6]. Spermatogenesis is a highly complex and organized process regulated by multi-genes. Spermatogonial stem cells (SSCs) and progenitor spermatogonia express self-renewal- and proliferation-associated genes such as Pou5f7[7], Zbtb16 (Plzf) [8], Taf4b[9], Neurog 3(Ngn3)[10], Lin28a[11, 12], Mir-21[13], Foxo1[14], Sox3[15], Mir-17 92 (Mirc7)[16], and Nanos2[17] to sustain the process of self-renewal and proliferation. Multiple genes are involved in sperm differentiation such as Kit[18], Cond2 [19], Soh/h7[20], Soh/h2[21], Sall4[22], and Stra8[23]to promote spermatogonial stem cells to differentiate into mature haploid spermatozoa. However, there are limited evidence-based data in the treatment of spermatogenesis disorders, and some drugs have obvious side effects.

In China, Traditional Chinese medicine (TCM) has been used to treat male infertility for nearly 2000 years, and increasingly appealing in surrounding areas and western countries. Holistic treatment is the essence and basic feature of TCM, and utilizing this theory for treating male infertility achieved satisfactory results[24, 25]. Sheng Jing Decoction (SJD) is one of traditional Chinese medicine prescription mainly be used to treat male infertility. SJD consists of several herbs such as Rehmannia glutinosa (Gaertn.) DC., Astragalus membranaceus (Fisch.) Bunge, Pseudostellaria heterophylla (Miq.) Pax, Dipsacus acaulis (A.Rich.) Napper, Lycium arenicolum Miers, Astragalus complanatus Bunge and Gleditsia sinensis Lam.. SJD has been applied in many hospitals in China, and patients' reaction is effective. However, the mechanism of action underlying the therapeutic effect of SJD is poorly understood. In this study, we used oligozoospermia model of ICR mice to verify the function of SJD and explore its mechanism. We identify that SJD can increase sperm concentration, sperm vitality, sperm motility and normal sperm morphology rate. To further confirm that SJD enhance sperm vitality and motility by keeping sperm mitochondria's function and sperm plasma membrane integrity. SJD can promote spermatogenesis by up-regulating the expression of the proliferation-associated gene Lin28a, and the differentiation-associated genes (Kit, Sohlh2 and Stra8).

\section{Methods}

\section{Experimental animals}

Male ICR mice were purchased from Shanghai Experimental Animal Center and kept in the Experimental Animal Building of Shanghai Institute of Family Planning Science (Clean grade, license No.:

SCXY(Shanghai)2018-0017). 


\section{Reagents and antibodies}

Cyclophosphamide (8H257F, Baxter International Inc, Illinois, USA). anti-TRA98 ( ab82527凶Abcam, Cambridge, MA,USA). anti- GATA4 ( ab84593, Abcam, Cambridge, MA,USA). anti-33-HSD (sc-515120, Sant Cruz, Oregon, USA ). anti-Ki67 (AF1738, Beyotime, Shanghai, China). TUNEL kit (C1086, Beyotime, Shanghai, China). LIVE/DEAD Sperm Viability kit (Thermo Fisher Scientific, Waltham, MA). PSA-FITC (012M4037V, Sigma, St. Louis, MOखUSA).

\section{Preparation of Sheng Jing Decoction}

Sheng Jing Decoction was combined with Rehmannia glutinosa (Gaertn.) DC., Astragalus membranaceus (Fisch.) Bunge, Pseudostellaria heterophylla (Miq.) Pax, Dipsacus acaulis (A.Rich.) Napper, Lycium arenicolum Miers, Astragalus complanatus Bunge and Gleditsia sinensis Lam.. All herbs pieces were purchased from Shanghai Wanshicheng TCM CO., Ltd, and made into SJD by TCM Pharmacy of Seventh People's Hospital of Shanghai University of TCM. Rehmannia glutinosa (Gaertn.) DC., Astragalus membranaceus (Fisch.) Bunge, Dipsacus acaulis (A.Rich.) Napper, Lycium arenicolum Miers, Astragalus complanatus Bunge and Gleditsia sinensis Lam. can be checked in "The Plant List" (www.theplantlist.org) and Pseudostellaria heterophylla (Miq.) Pax is available in MPNS (http://mpns.kew.org) The details of herbs can be checked in Supplemental Table 1.

\section{Experimental design}

The oligozoospermia model mice were constructed by cyclophosphamide inducing. Cyclophosphamide was dissolved in PBS and injected intraperitoneally (ip) to the mice $(60 \mathrm{mg} / \mathrm{kg})$, once in a day for a period of 5 days. High $(33 \mathrm{~g} / \mathrm{kg})$, medium $(16.5 \mathrm{~g} / \mathrm{kg})$ and low $(8.25 \mathrm{~g} / \mathrm{kg})$ doses of SJD were given orally to mice, once in a day for a period of 35 days. The medium dose is converted from the therapeutic dose of human. Body weights were measured every week. Testes were quickly dissected out and weighed. One half of testes were collected and stored at $-70 \circ \mathrm{C}$ for real-time RT-PCR analysis, and the other half of testes were fixed in Bouin's fixative (0.2\% picric acid/2\% ( $/ \mathrm{v})$ formaldehyde in PBS) for histological evaluation.

\section{Sperm motility and count}

The epididymis was placed in $1 \mathrm{ml}$ normal saline, and then a deep hole was cut in each caudate nucleus with micro scissors. Sperm was allowed to release for 5 minutes in an incubator with a temperature of $34^{\circ} \mathrm{C}$ and $5 \% \mathrm{CO}$. The sperm suspension was incubated in $\mathrm{CO} 2$ incubator for $30 \mathrm{~min}$ and a drop of sperm suspension was uniformly smeared on clean glass slide. Sperm motility and count were analyzed by CASA system and bright-field microscopy (BX43 Olympus, Tokyo, Japan).

\section{Immunohistochemistry}

Immunohistochemistry was performed according to standard procedures. The primary antibodies antiTRA98 (1: 200), anti- GATA4 (1: 200), anti-3ß-HSD (1: 100) and anti- Ki67(1: 100), ABC Kit (Vector, USA) 
and DAB (ZSGB-bio, China) were used for staining. The results were obtained by fluorescence microscope (BX51 Olympus, Tokyo, Japan) and Color digital camera (DFC425 Leica, Wetzlar, Germany).

\section{Real-time RT-PCR analysis}

Total RNA was prepared using TRIzol (Sigma, USA) and was reversely transcribed into cDNA with the QuantiNova Reverse Transcription Kit (Qiagen, Dusseldorf, Germany). Real-time quantitative PCR was performed using a CFX96TM Real-time system (Bio-Rad, USA) and SYBR Premix Ex Taq (TakaRa, Dalian, China) according to the manufacturer's instruction. Raw data were normalized to the internal GAPDH and presented as relative expression level calculated by $2 \triangle \triangle \mathrm{Ct}$ method. Primers pairs were as following: Lin28a:5'-GGTGGTGTGTTCTGTATTGGGA-3' and 5'-AGTTGTAGCACCTGTCTCCCTTTG-3'. Kit. 5'GGGCTAGCCAGAGACATCAG-3' and 5'-AGGAGAAGAGCTCCCAGAGG-3'. Soh/h2: 5'-TCTCAGCCACATCAC AGAGG-3' and 5'-GGGGACGCGAGTCTTATACA-3'. Stra8: 5'-ACCCTG GTAGGGCTCTTCAA-3' and 5'GACCTCCTCTAAGCTGTTGGG-3'.

\section{JC-1 staining}

JC-1 staining kit was purchased from Beyotime (Shanghai, China), Equal volume sperm and JC-1 staining working fluid were mixed. Incubated at $37^{\circ} \mathrm{C}$ with $5 \% \mathrm{CO} 2$ for $30 \mathrm{~min}$. Then the sperm were washed twice with JC-1 staining buffer. The results were observed under fluorescence microscope (BX51 Olympus, Tokyo, Japan).

\section{Sperm plasma membrane integrity test}

The Sperm $(1 \times 10 / \mathrm{mL})$ suspension was added syBR-14 and PI dye kit(Thermo Fisher Scientific, Waltham, $M A)$ successively. Mixed and incubated in dark for 5 min. After washing, stained sperm were observed by fluorescence microscope (BX51 Olympus, Tokyo, Japan).

\section{Statistics and data analyses}

Data are expressed as the mean \pm SEM, and statistical evaluation was performed using the Student's ttest for independent groups. Values of $p<0.05$ were considered statistically significant.

\section{Results}

\section{SJD can accelerate recovery from impairment caused by cyclophosphamide inducing in the whole body and testicular tissue}

To investigate the function of SJD, we constructed oligozoospermia model of ICR mice induced by injecting intraperitoneally (ip) with $60 \mathrm{mg} / \mathrm{kg}$ dose of cyclophosphamide[26]. At the same time of modeling, high, medium and low doses of SJD were given orally for treatment respectively. We measured the body weight of mice after ip cyclophosphamide every week and testis weight for SJD treatment 35 days. The high, medium and low doses of SJD all can speed up the recovery from weight loss of whole 
body by cyclophosphamide inducing (Fig. 1A). The relative weight of the testes of oligozoospermia model mice significantly increased after SJD treatment (Fig. 1B). H\&E staining of analysis of fresh testicular tissue found that testicular tissue was severely damaged by cyclophosphamide inducing, and SJD is helpful to restoration from the damage of testicular tissue (Fig. 1C). Testicular tissue cells mainly include Sertoli cells, germ cells Leydig cells, we can determine them by their respective molecular marker (GATA4, TRA98 and 3ß-HSD). Immunohistochemistry data showed sertoli cells and germ cells were remarkably damaged oligozoospermia model mice, and could be partly recovered by SJD treatment (Fig. 1C). Whereas Leydig cells had no significant differences during different groups (Fig. 1C).

\section{SJD can partly restore the defects of sperm quantity and quality in oligozoospermia model mice}

To evaluate the concentration, vitality and motility of sperm by computer-aided sperm analysis (CASA). CASA reports revealed sperm concentration, vitality and motility of oligozoospermia model mice without SJD treatment were significantly decreased compare with control group, whereas it had a significant improvement of sperm concentration, vitality and motility after SJD treatment (Fig. 2A, B and C). Normal sperm morphology rate was markedly increase in oligozoospermia model mice by SJD treatment compared to without SJD treatment (Fig. 2D).

\section{SJD can promote spermatogenesis and up-regulate the expression of the proliferation-associated gene Lin28a and the differentiation-associated genes (Kit, Sohlh2 and Stra8)}

The above data showed SJD can significantly increase the number of sertoli cells and germ cells of oligozoospermia model mice. In addition, Ki67 staining analysis also confirmed that SJD has the function of promoting proliferation of testicular tissue cells in oligozoospermia model mice (Fig. 3A). To further investigate the mechanism of SJD, we examined the mRNA expression of the proliferationassociated gene Lin28a, and the differentiation-associated genes (Kit, Soh/h2 and Stra8) by performing quantitative RT-PCR. The results suggested that the expression of genes Lin28a,Kit, Sohlh2 and Stra8 have no difference in oligozoospermia model mice without SJD treatment compare with normal mice, but significantly up-regulated in oligozoospermia model mice by SJD treatment (Fig. 3B, C, D and E).

\section{SJD has the effect of sustaining sperm mitochondrial membrane potential (MMP) and sperm plasma membrane integrity}

Sperm mitochondrial membrane potential (MMP) and sperm plasma membrane integrity were two important factors involved in sperm motility. MMP was tested and analyzed by JC-1 staining. The results showed MMP was remarkably decreased by cyclophosphamide inducing, whereas SJD treatment could offset the loss of MMP (Fig 4A). SYBR-14/PI double staining analysis showed that sperm plasma membrane integrity was less impaired in oligozoospermia model mice by SJD treatment compared with without SJD treatment (Fig. 4 B).

\section{Discussion}


In recent years, male infertility has been attracting more attention from public with the decline of semen

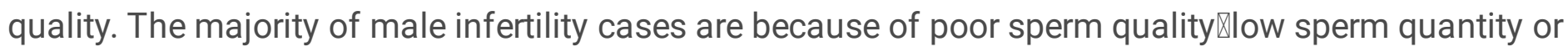
both $[27,28]$. There are many factors leading to the male infertility including abnormal spermatogenesis[29, 30]; anomalies or obstruction of reproductive tract [31]; abnormal sexual and ejaculatory functions [32]; and inadequate sperm motility[33], imbalance in hormone levels[34], and genetic defects[4]. In addition, idiopathic infertility accounts for $30 \%$ of infertile men [35]. Although a great progression has been made in treating male infertility, including intrauterine insemination, in vitro fertilization (IVF) and even intracytoplasmic sperm injection (ICSI), but these treatments have disadvantages such as unsatisfactory efficacy, strong invasiveness, high risk, or excessive cost. Therefore, it is necessary to seek effective natural therapy to increase fertility. TCM has been used to treat male infertility in China for a very long time and has now been using increasingly in neighboring and western countries [24, 36]. SJD, as a traditional Chinese medicine prescription, has been used to treat male infertility in many Chinese hospital and helped many men with fertility problems achieve clinical pregnancy. However, its biological function and the relevant mechanism is poorly understood.

To investigate the role of SJD in treating male infertility, we established the oligozoospermia model of ICR mice by cyclophosphamide inducing. Cyclophosphamide caused temporary impairment of normal male reproductive system by low dose treatment. The weight of the whole body and testis significantly decreased after cyclophosphamide inducing (Fig. 1A and B), and testicular tissue was severely damaged (Fig. 1C). There is a great impairment of sperm and its fertilizing ability in oligozoospermia mice model (Fig. 2). At the same time of modeling, SJD were given orally for treatment. SJD is helpful to restoration from the damage of testicular tissue (Fig. 1C). It had a significant increase of sperm concentration, vitality and motility and normal sperm morphology rate after SJD treatment in oligozoospermia model mice (Fig. 2), which verified SJD treats male infertility mainly by enhancing sperm quantity and quality.

Male fertility is maintained through complex cellular and molecular interactions, which ensure that spermatogonial stem cells (SSCs) self-renewal and differentiation into mature sperm[6].

Spermatogenesis is a highly organized and complex process, which can continuously produce millions of haploid sperm in the whole adult male life, and keep the complete genome and appropriate epigenome from generation to generation[30,37]. Our data showed that the expression of sertoli cells marker GATA4[38] and germ cells marker TRA98[39] increased by SJD treatment in oligozoospermia model mice (Fig. 1C), and sperm concentration and vitality significantly increased by SJD treatment (Fig. 2A and B). In addition, SJD has the function of enhancing the ability of proliferation of testicular tissue cells in oligozoospermia model mice (Fig. 3A). These data demonstrated that SJD plays promoting roles on selfrenewal, proliferation and differentiation process during which diploid SSCs produce haploid spermatozoa.

Spermatogonium is the most primitive spermatogonia in the testis, which plays an important role in maintaining sperm production. Through self-renewal and continuous daughter spermatogonia differentiation, transmitting genetic information to the next generation. Self-renewal- and proliferationassociated genes such as Pou5f1, Lin28a, Mir-21, Mir-17 92 (Mirc1), Foxo1, Nanos2, Neurog 3(Ngn3), 
Sox3, Taf4b, and Zbtb16 (Plzf) to maintain the capacity for self-renewal and proliferation of SSCs and progenitor spermatogonia[7-17]. In the process of spermatogonia differentiation, undifferentiated spermatogonia downregulate self-renewal related genes and upregulate differentiation related genes such as Sohlh1, Sohlh2, Stra8, Kit, Ccnd2 and Sall4 to promote spermatogonial stem cells to differentiate into mature haploid spermatozoa[18-23]. Our studies indicated the expression of proliferation-associated gene Lin28a, and differentiation-associated genes Kit, Soh/h2 and Stra8 significantly up-regulated in oligozoospermia model mice by SJD treatment (Fig. 3B, C, D and E). The results further verified that SJD promoted the spermatogenesis by upregulating the proliferation-associated gene Lin28a, and differentiation-associated genes Kit, Sohlh2 and Stra8.

Sperm motility is an important factor involved in the overall sperm quality, critical to ensure fertilization success[40]. The mitochondrion is the major energy provider to sustain sperm motility. Previous studies focused on the time regulation of mitochondrial membrane potential (MMP) on sperm motility. MMP can be used as a potential regulator and indicator of sperm motility, which is directly related to male fertility[41-43]. Our data showed that SJD returned MMP of sperm impaired by cyclophosphamide inducing to normal (Fig. 4A). These data demonstrated that SJD play an important role to sustain mitochondrial function and sperm motility. As a dynamic platform, many components of membrane participate in cell motility process, including force generation, adhesion, signal transmission and regulation[44]. Sperm plasma membrane integrity plays a crucial role in sperm motility and fertilization $[45,46]$. Our results showed that sperm plasma membrane integrity was less impaired in oligozoospermia model mice by SJD treatment compared to without SJD treatment (Fig. 4B). These data provided strong evidences that SJD is an important factor involved in supporting sperm plasma membrane integrity and functions.

\section{Conclusions}

In summary, SJD, as a traditional Chinese medicine prescription, has been popularly used to treat male infertility in many Chinese hospital. SJD can increase sperm concentration, sperm vitality, sperm motility and normal sperm morphology rate, to support sperm quantity and quality. SJD has the effect of promoting the spermatogenesis, and further demonstrated that it up-regulates the proliferationassociated gene Lin28a, and differentiation-associated genes Kit, Soh/h2 and Stra8. In addition, our data confirmed SJD increases sperm motility by sustaining MMP and sperm plasma membrane integrity.

\section{Abbreviations}

SJD, Sheng Jing Decoction; TCM, Traditional Chinese medicine; SSCs, Spermatogonial stem cells; CASA, Computer-aided sperm analysis; MMP, Sperm mitochondrial membrane potential.

\section{Declarations}

\section{Ethics approval and consent to participate}


All mice were purchased from Shanghai Experimental Animal Center冈and the experiments were approved by the Research Ethics Committee, Seventh People's Hospital of Shanghai University of TCM (Shanghai, China).

\section{Consent to publish}

All the authors were concerned and agreed to publish before the submission.

\section{Availability of data and materials}

Details of data mining, selection, extraction and assessment carried out to support the findings of this study are available from the corresponding author upon request.

\section{Competing interests}

The authors declare that there are no competing interests regarding the publication of this paper.

\section{Funding}

This work was supported by the construction of key disciplines of Traditional Chinese Medicine From Health Commission of Pudong New Area (NO. : PDZY-2018-0608).

\section{Authors' contributions}

YG and TF performed molecular biology experiments, in vivo experiments and statistics analysis; LP, MJM and HWJ conducted the immunohistochemistry staining and CASA analysis. MR, GSS and YQY performed oligozoospermia model mice feeding and measurement; SJM and YG designed the study and wrote the manuscript; All authors have read and approved the final manuscript.

\section{Acknowledgements}

We kindly thank the Health Commission of Pudong New Area.

\section{References}

1. Mascarenhas MN, Flaxman SR, Boerma T, Vanderpoel S, Stevens GA. National, regional, and global trends in infertility prevalence since 1990: a systematic analysis of 277 health surveys. PLoS Med. 2012; 9(12): e1001356.

2. PJ R. WHO manual for the standardized investigation, diagnosis and management of the infertile male. Cambridge: Cambridge University Press. 2000 5th ed: 1-5.

3. Barratt CLR, Bjorndahl L, De Jonge CJ, Lamb DJ, Osorio Martini F, McLachlan R, et al. The diagnosis of male infertility: an analysis of the evidence to support the development of global WHO guidancechallenges and future research opportunities. Hum Reprod Update. 2017; 23(6): 660-680. 
4. Krausz C, Riera-Escamilla A. Genetics of male infertility. Nature reviews Urology. 2018; 15(6): 369384.

5. Tournaye $\mathrm{H}$, Krausz C, Oates RD. Novel concepts in the aetiology of male reproductive impairment. Lancet Diabetes Endocrinol. 2017; 5(7): 544-553.

6. Kubota H, Brinster RL. Spermatogonial stem cells. Biol Reprod. 2018; 99(1): 52-74.

7. Pesce $M$, Wang $X$, Wolgemuth DJ, Scholer $H$. Differential expression of the Oct-4 transcription factor during mouse germ cell differentiation. Mech Dev. 1998; 71(1-2): 89-98.

8. Buaas FW, Kirsh AL, Sharma M, McLean DJ, Morris JL, Griswold MD, et al. Plzf is required in adult male germ cells for stem cell self-renewal. Nature genetics. 2004; 36(6): 647-652.

9. Falender AE, Freiman RN, Geles KG, Lo KC, Hwang K, Lamb DJ, et al. Maintenance of spermatogenesis requires TAF4b, a gonad-specific subunit of TFIID. Genes \& development. 2005; 19(7): 794-803.

10. Nakagawa T, Nabeshima Y, Yoshida S. Functional identification of the actual and potential stem cell compartments in mouse spermatogenesis. Developmental cell. 2007; 12(2): 195-206.

11. Tong MH, Mitchell D, Evanoff R, Griswold MD. Expression of Mirlet7 family microRNAs in response to retinoic acid-induced spermatogonial differentiation in mice. Biol Reprod. 2011; 85(1): 189-197.

12. Zheng K, Wu X, Kaestner KH, Wang PJ. The pluripotency factor LIN28 marks undifferentiated spermatogonia in mouse. BMC Dev Biol. 2009; 9: 38.

13. Niu Z, Goodyear SM, Rao S, Wu X, Tobias JW, Avarbock MR, et al. MicroRNA-21 regulates the selfrenewal of mouse spermatogonial stem cells. Proceedings of the National Academy of Sciences of the United States of America. 2011; 108(31): 12740-12745.

14. Goertz MJ, Wu Z, Gallardo TD, Hamra FK, Castrillon DH. Foxo1 is required in mouse spermatogonial stem cells for their maintenance and the initiation of spermatogenesis. The Journal of clinical investigation. 2011; 121(9): 3456-3466.

15. Laronda MM, Jameson JL. Sox3 functions in a cell-autonomous manner to regulate spermatogonial differentiation in mice. Endocrinology. 2011; 152(4): 1606-1615.

16. Tong MH, Mitchell DA, McGowan SD, Evanoff R, Griswold MD. Two miRNA clusters, Mir-17-92 (Mirc1) and Mir-106b-25 (Mirc3), are involved in the regulation of spermatogonial differentiation in mice. Biol Reprod. 2012; 86(3): 72.

17. Zhou Z, Shirakawa T, Ohbo K, Sada A, Wu Q, Hasegawa K, et al. RNA Binding Protein Nanos2 Organizes Post-transcriptional Buffering System to Retain Primitive State of Mouse Spermatogonial Stem Cells. Developmental cell. 2015; 34(1): 96-107.

18. Schrans-Stassen BH, van de Kant HJ, de Rooij DG, van Pelt AM. Differential expression of c-kit in mouse undifferentiated and differentiating type A spermatogonia. Endocrinology. 1999; 140(12): 5894-5900.

19. Beumer TL, Roepers-Gajadien HL, Gademan IS, Kal HB, de Rooij DG. Involvement of the D-type cyclins in germ cell proliferation and differentiation in the mouse. Biol Reprod. 2000; 63(6): 1893- 
1898.

20. Ballow D, Meistrich ML, Matzuk M, Rajkovic A. Sohlh1 is essential for spermatogonial differentiation. Developmental biology. 2006; 294(1): 161-167.

21. Hao J, Yamamoto M, Richardson TE, Chapman KM, Denard BS, Hammer RE, et al. Sohlh2 knockout mice are male-sterile because of degeneration of differentiating type A spermatogonia. Stem cells. 2008; 26(6): 1587-1597.

22. Gely-Pernot A, Raverdeau M, Teletin M, Vernet N, Feret B, Klopfenstein M, et al. Retinoic Acid Receptors Control Spermatogonia Cell-Fate and Induce Expression of the SALL4A Transcription Factor. PLoS Genet. 2015; 11(10): e1005501.

23. Endo T, Romer KA, Anderson EL, Baltus AE, de Rooij DG, Page DC. Periodic retinoic acid-STRA8 signaling intersects with periodic germ-cell competencies to regulate spermatogenesis. Proceedings of the National Academy of Sciences of the United States of America. 2015; 112(18): E2347-2356.

24. Zhou SH, Deng YF, Weng ZW, Weng HW, Liu ZD. Traditional Chinese Medicine as a Remedy for Male Infertility: A Review. The world journal of men's health. 2019; 37(2): 175-185.

25. Jiang D, Coscione A, Li L, Zeng BY. Effect of Chinese Herbal Medicine on Male Infertility. Int Rev Neurobiol. 2017; 135: 297-311.

26. Elangovan N, Chiou TJ, Tzeng WF, Chu ST. Cyclophosphamide treatment causes impairment of sperm and its fertilizing ability in mice. Toxicology. 2006; 222(1-2): 60-70.

27. Bensdorp AJ, Cohlen BJ, Heineman MJ, Vandekerckhove P. Intra-uterine insemination for male subfertility. The Cochrane database of systematic reviews. 2007(4): CD000360.

28. Alrabeeah K, Yafi F, Flageole C, Phillips S, Wachter A, Bissonnette F, et al. Testicular sperm aspiration for nonazoospermic men: sperm retrieval and intracytoplasmic sperm injection outcomes. Urology. 2014; 84(6): 1342-1346.

29. de Rooij DG. The nature and dynamics of spermatogonial stem cells. Development. 2017; 144(17): 3022-3030.

30. Fayomi AP, Orwig KE. Spermatogonial stem cells and spermatogenesis in mice, monkeys and men. Stem Cell Res. 2018; 29: 207-214.

31. Jensen CFS, Ostergren P, Dupree JM, OhI DA, Sonksen J, Fode M. Varicocele and male infertility. Nature reviews Urology. 2017; 14(9): 523-533.

32. Lotti F, Maggi M. Sexual dysfunction and male infertility. Nature reviews Urology. 2018; 15(5): 287307.

33. Zhu X, Xie T, Zhan X, Liu L, Yang X, Xu X, et al. Association of seminal angiotensinogen with sperm motility and morphology in male infertility. Andrologia. 2019; 51(11): e13421.

34. Ohlander SJ, Lindgren MC, Lipshultz LI. Testosterone and Male Infertility. The Urologic clinics of North America. 2016; 43(2): 195-202.

35. Jungwirth A, Giwercman A, ournaye H, Diemer T, Kopa Z, Dohle G. European Association of Urology guidelines on male infertility: the 2012 update. European urology. 2012; 62: 324-332. 
36. Zhang Z, Yang J, Kong T, Wang C, Ning P, Chen X, et al. Traditional Chinese Medicine syndrome elements of male infertility revealed by latent tree model analysis. J Tradit Chin Med. 2018; 38(6): 926-935.

37. Komeya M, Ogawa T. Spermatogonial stem cells: Progress and prospects. Asian journal of andrology. 2015; 17(5): 771-775.

38. Liang J, Wang N, He J, Du J, Guo Y, Li L, et al. Induction of Sertoli-like cells from human fibroblasts by NR5A1 and GATA4. eLife. 2019; 8.

39. Tanaka H, Pereira LA, Nozaki M, Tsuchida J, Sawada K, Mori H, et al. A germ cell-specific nuclear antigen recognized by a monoclonal antibody raised against mouse testicular germ cells. Int $\mathrm{J}$ Androl. 1997; 20(6): 361-366.

40. Harlev A, Henkel R, Samanta L, Agarwal A. Ritalinic Acid Stimulates Human Sperm Motility and Maintains Vitality In Vitro. The world journal of men's health. 2020; 38(1): 61-67.

41. Piomboni P, Focarelli R, Stendardi A, Ferramosca A, Zara V. The role of mitochondria in energy production for human sperm motility. Int J Androl. 2012; 35(2): 109-124.

42. Barbagallo F, La Vignera S, Cannarella R, Aversa A, Calogero AE, Condorelli RA. Evaluation of Sperm Mitochondrial Function: A Key Organelle for Sperm Motility. J Clin Med. 2020; 9(2).

43. Agnihotri SK, Agrawal AK, Hakim BA, Vishwakarma AL, Narender T, Sachan R, et al. Mitochondrial membrane potential (MMP) regulates sperm motility. In Vitro Cell Dev Biol Anim. 2016; 52(9): 953960.

44. Keren K. Cell motility: the integrating role of the plasma membrane. Eur Biophys J. 2011; 40(9): 10131027.

45. Lucio CF, Brito MM, Angrimani D, Belaz K, Morais D, Zampieri D, et al. Lipid composition of the canine sperm plasma membrane as markers of sperm motility. Reprod Domest Anim. 2017; 52 Suppl 2: 208213.

46. Schuh K, Cartwright EJ, Jankevics E, Bundschu K, Liebermann J, Williams JC, et al. Plasma membrane $\mathrm{Ca} 2+$ ATPase 4 is required for sperm motility and male fertility. The Journal of biological chemistry. 2004; 279(27): 28220-28226.

\section{Figures}


A

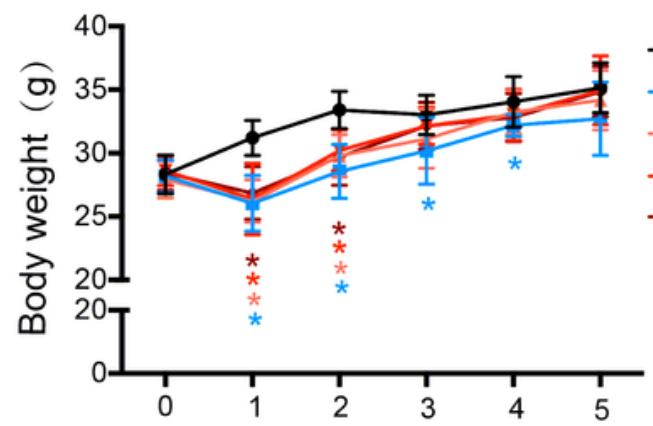

Times after cyclophosphamide injection (weeks)

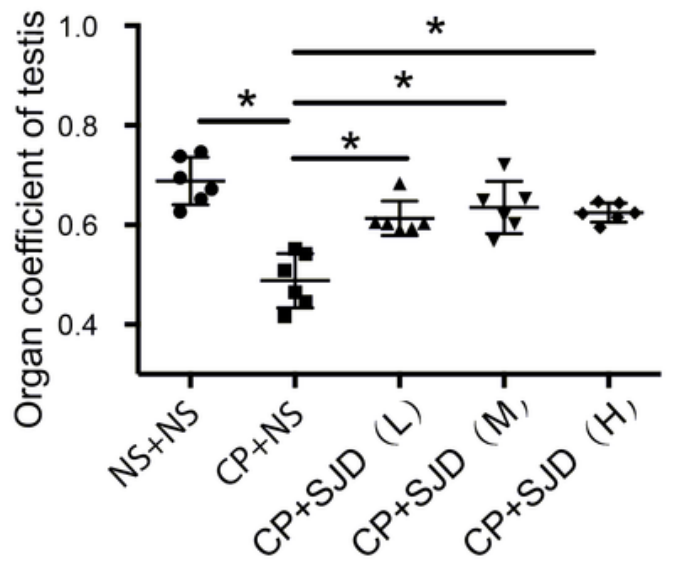

C
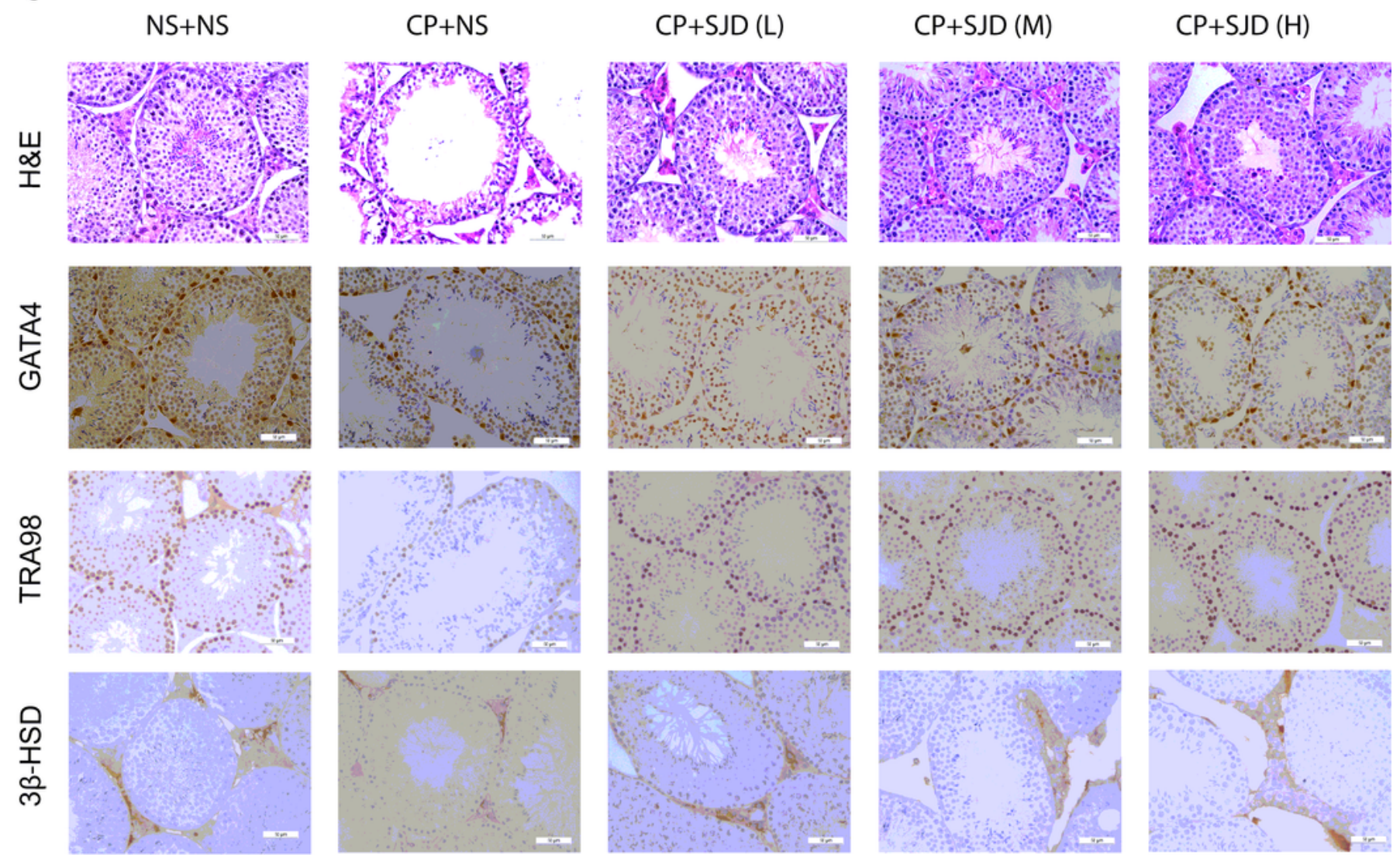

\section{Figure 1}

SJD promotes recovery from impairment caused by cyclophosphamide inducing. (A) Effect of cyclophosphamide and SJD treatment on the whole body. Body weights were obtained after 1 st -5 th weeks of last injection. Data were means \pm SEM. $n=6$ in each group, ${ }^{*} p<0.05$. (B) Effect of cyclophosphamide and SJD treatment on the relative weight of the testes. Data were collected after 5th weeks of last injection. Data were means \pm SEM. $n=6$ in each group, ${ }^{*} p<0.05$. (C) HखE and 
immunohistochemistry analysis of GATA4, TRA98 and 3ß-HSD expression in testicular tissues. Scale bar, $50 \mu \mathrm{m}$. NS: normal saline, CP: cyclophosphamide, SJD(L): low doses of Sheng Jing Decoction, SJD(M): medium doses of Sheng Jing Decoction, SJD $(H)$ : high doses of Sheng Jing Decoction.

Figure 2

A

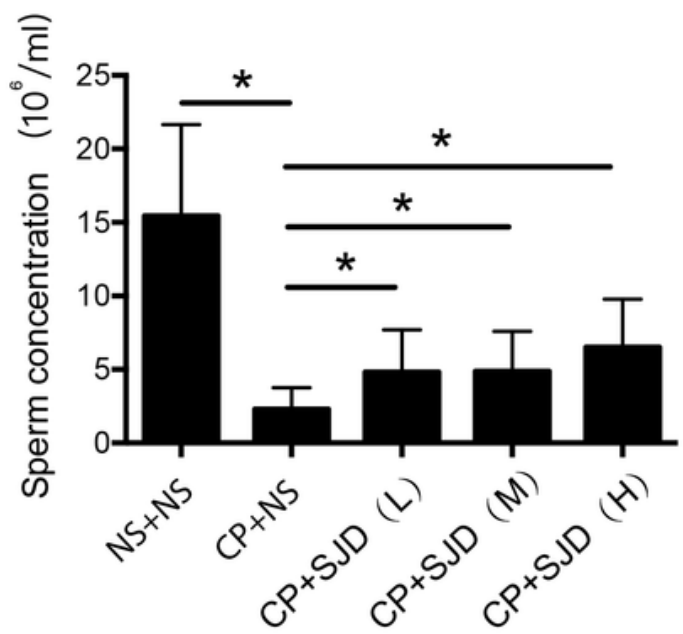

C

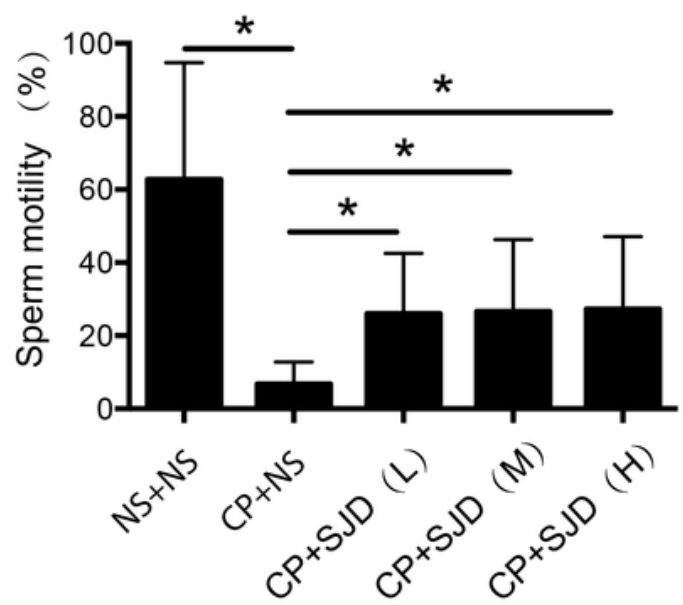

B

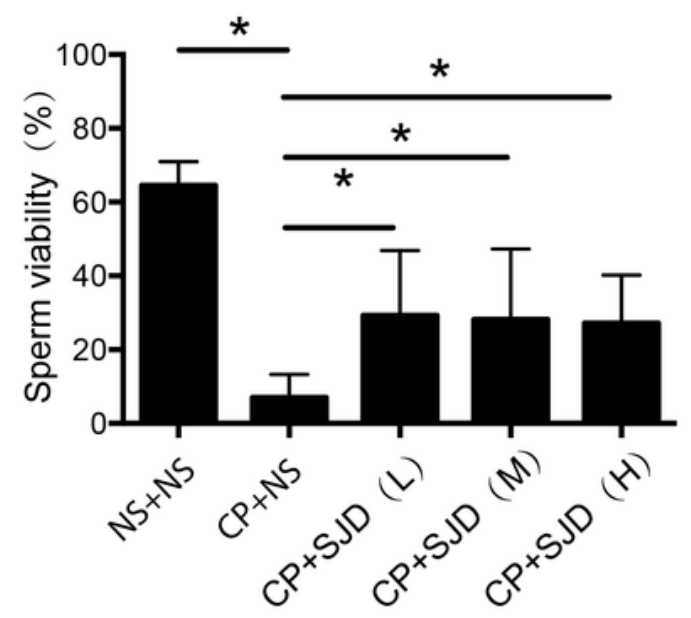

D

@

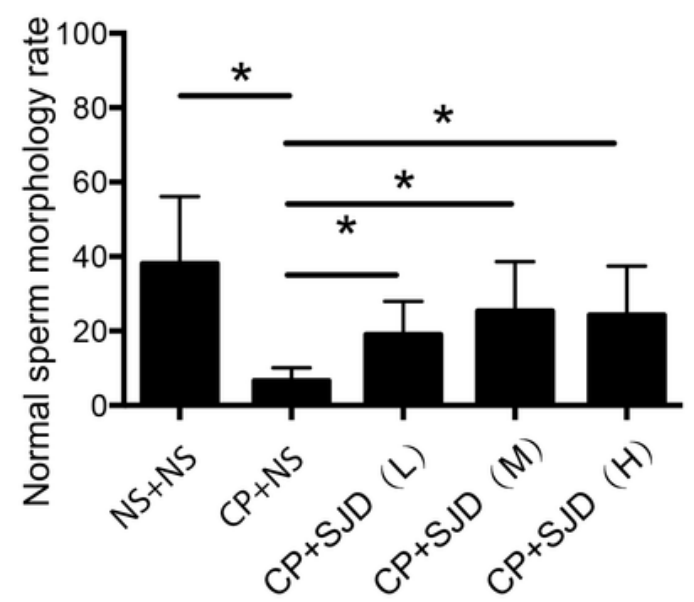

Figure 2

SJD increases sperm quantity and quality of oligozoospermia model mice. (A), (B)and (C): The concentration, vitality and motility of sperm by computer-aided sperm analysis (CASA). (D) Normal sperm 
morphology rate was determined by PAP staining. Data were means \pm SEM. $n=6$ in each group, ${ }^{*} p<0.05$. Figure 3

A
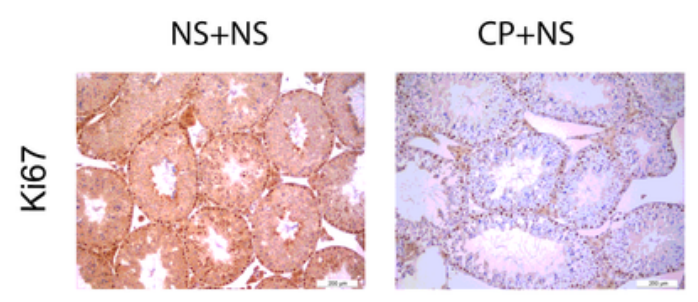

$\mathrm{CP}+\mathrm{SJT}(\mathrm{L})$

$\mathrm{CP}+\mathrm{SJT}$ (M)

$\mathrm{CP}+\mathrm{SJT}(\mathrm{H})$
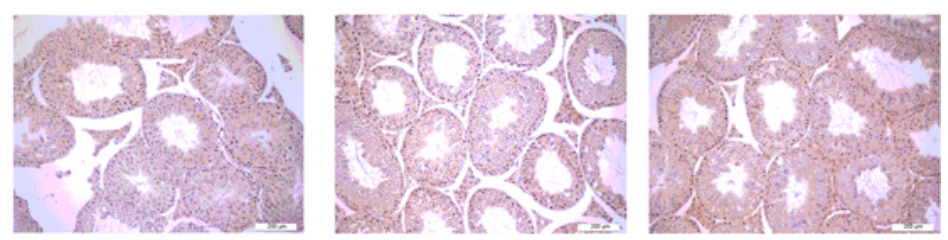

B

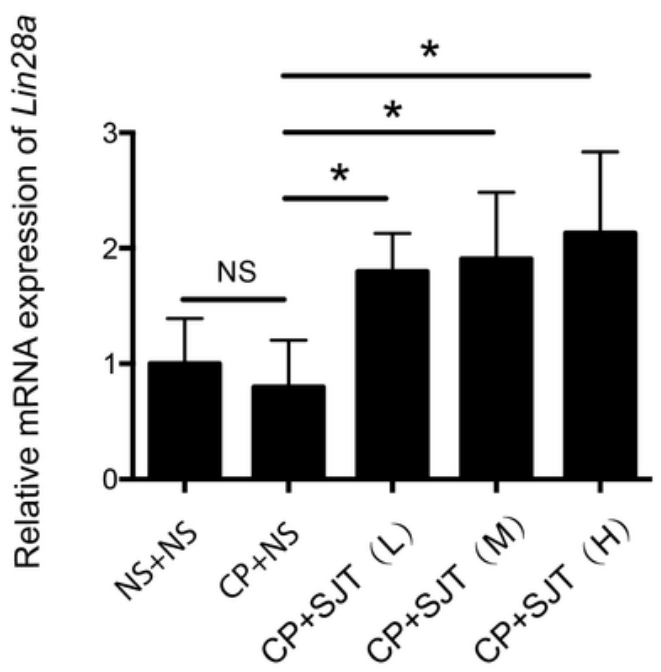

C

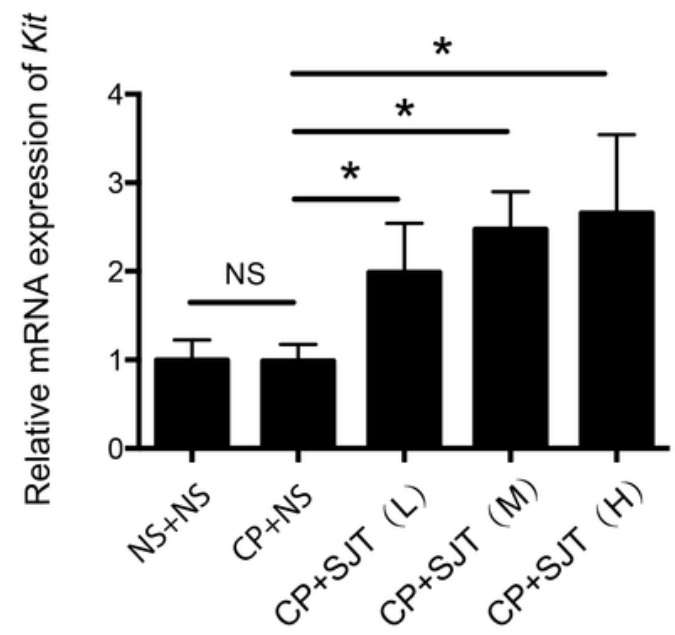

D

E
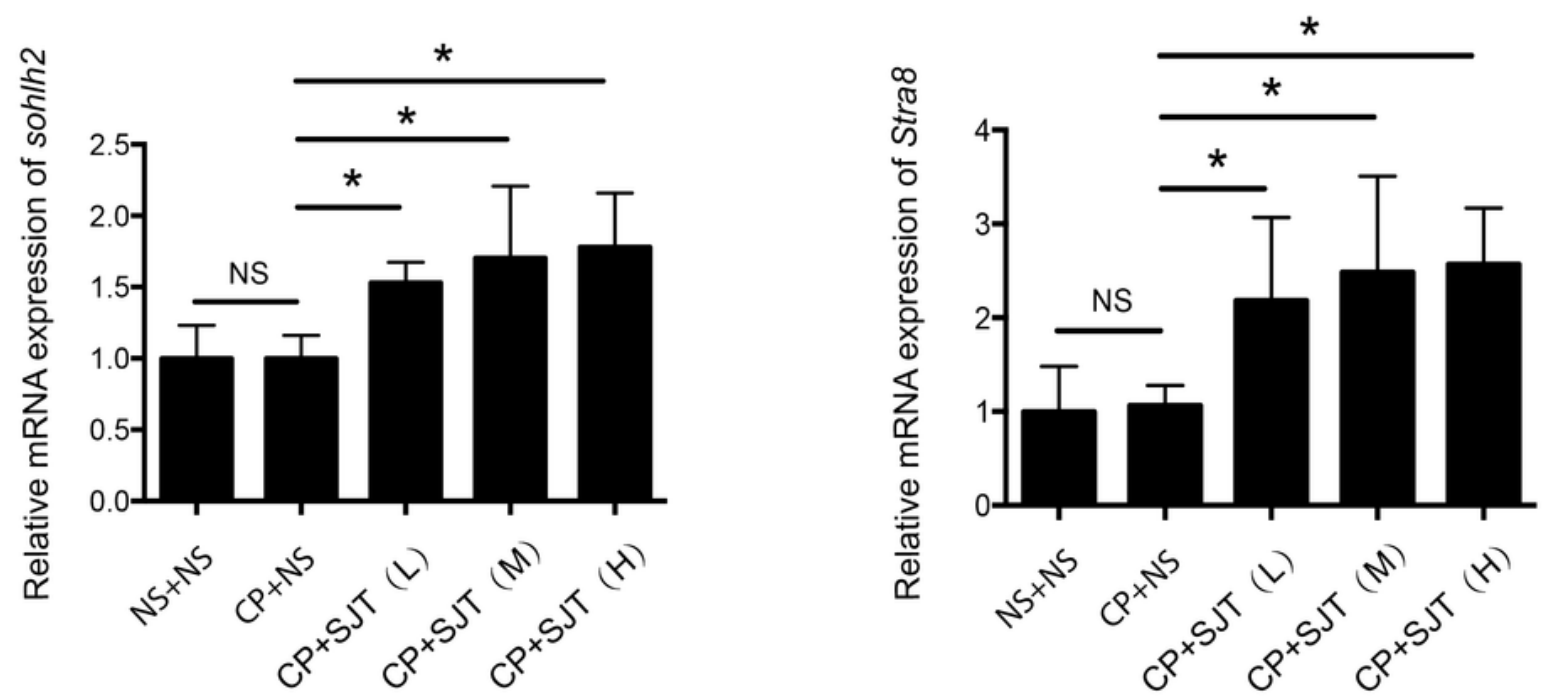

Figure 3

SJD promotes spermatogenesis and up-regulate genes Lin28a, Kit, Sohlh2 and Stra8. (A): The proliferation ability of testicular tissue cells was reflected by Ki-67 staining. (B), (C), (D) and (E): Real-time RT-PCR analysis of Lin28a, Kit, Sohlh2 and Stra8 in oligozoospermia model mice after SJD treatment and control group mice. Data were means \pm SEM. $n=6$ in each group, ${ }^{*} p<0.05$. 
Figure 4

A
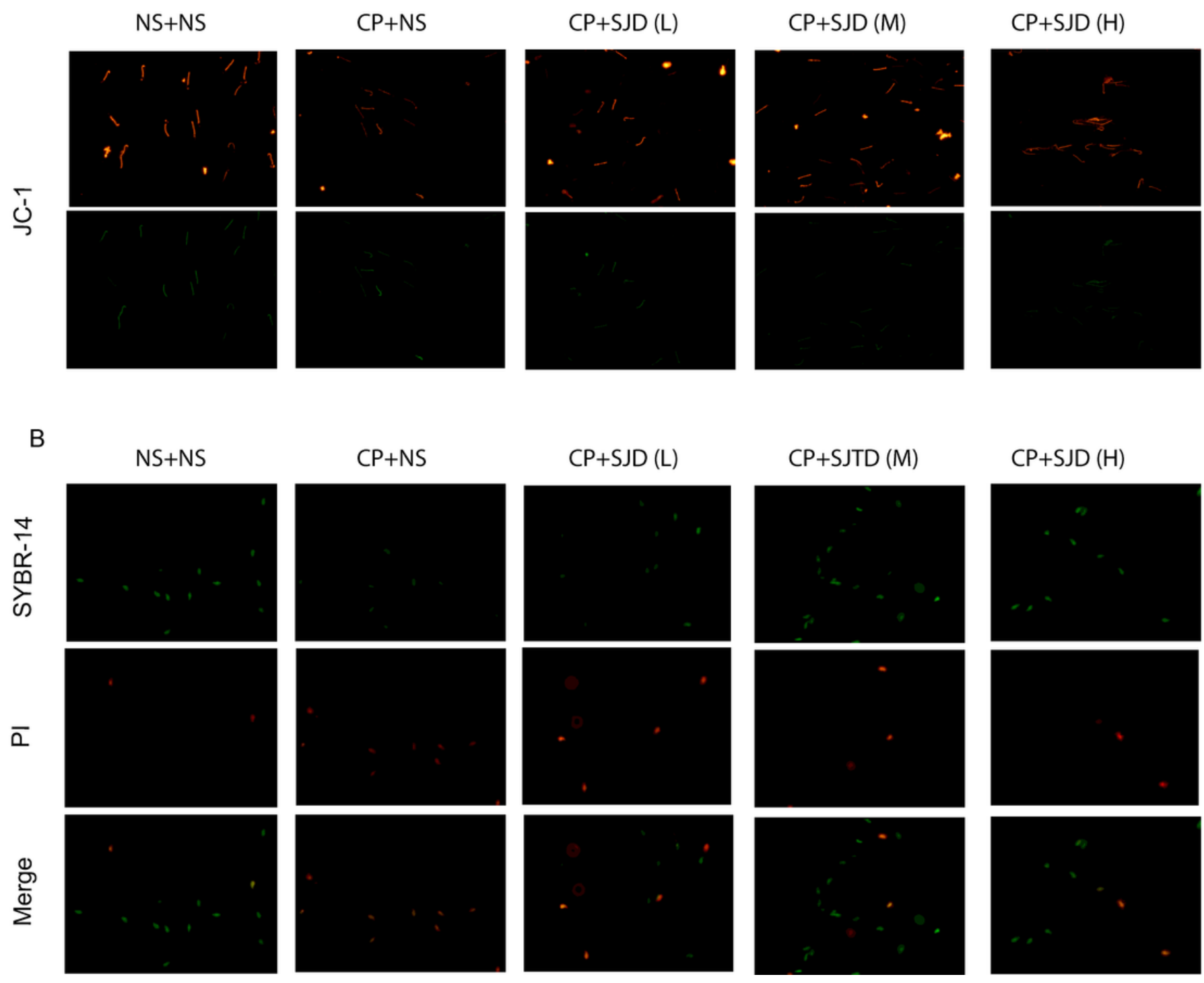

Figure 4

Effect of SJD treatment on sperm mitochondrial membrane potential (MMP) and sperm plasma membrane integrity (A) MMP was tested and analyzed by JC-1 in oligozoospermia model mice after SJD treatment and control group mice. (B) Sperm plasma membrane integrity was determined by SYBR-14/PI double staining in oligozoospermia model mice after SJD treatment and control group mice.

\section{Supplementary Files}

This is a list of supplementary files associated with this preprint. Click to download.

- SupplementalTable1.xlsx 